\title{
EFFECT OF A BIOACTIVE CALCIUM ALKALI ORTHOPHOSPHATE BONE GRAFTING MATERIAL AS COMPARED TO TRICALCIUM PHOSPHATE ON OSTEOGENESIS AFTER SINUS FLOOR AUGMENTATION IN PATIENTS
}

\author{
Hana-Ayad Ensir ${ }^{1,2}$, Tom Knauf ${ }^{1,3}$, Doaa Adel-Khattab ${ }^{1,4}$, Alina Bednarek ${ }^{1}$, Imran Tariq ${ }^{5,6}$, \\ Georg Berger $^{7}$, Renate Gildenhaar ${ }^{7}$, Michael Stiller ${ }^{1,8}$, $\underline{\text { Christine Knabe }^{1 \S}}$ \\ ${ }^{1}$ Dept. of Experimental Orofacial Medicine, School of Dental Medicine, Philipps University, Marburg, Germany, \\ ${ }^{2}$ Aviation and Submarine Medical Centre, Maetiga Hospital, Tripoli, Libya \\ ${ }^{3}$ Dept. of Orthopedics and Traumatology, Philipps University, Marburg, Germany \\ ${ }^{4}$ Dept. of Periodontology, School of Dentistry, Ain Shams University, Cairo, Egypt \\ ${ }^{5}$ Dept. of Pharmaceutics and Biopharmaceutics, Philipps University, Marburg, Germany \\ ${ }^{6}$ University College of Pharmacy, University of the Punjab, Lahore 54000, Pakistan \\ ${ }^{7}$ Federal Institute for Materials Research and Testing, Laboratory of Biomaterials, Berlin, Germany \\ ${ }^{8}$ Dept. of Prosthodontics, School of Dental Medicine, Philipps University, Marburg, Germany
}

${ }^{\S}$ Authors have contributed equally.

Corresponding Author: Christine Knabe, DDS, Ph.D.

Tel: +49(0)6421 5863600; Fax: +49(0)6421 5863606

E-mail: knabec@med.uni-marburg.de

https://doi.org/10.34107/BiomedSciInstrum.57.0101

\begin{abstract}
Sinus floor augmentation (SFA) has become a well-recognized procedure for site development in the atrophic posterior maxilla prior to dental implant placement. There has been an increasing search for relatively rapidly resorbable bone substitutes that enhance bone formation and facilitate bone regeneration with complete replacement by functional bone tissue. This has led to the development of a glassy crystalline silica-containing calcium alkali orthophosphate (Si-CAOP). The specific aim of this study was to assess histologically the effect of this grafting material on osteogenesis and osteogenic marker expression and to compare this effect to that of $\beta$-tricalcium phosphate ( $\beta$-TCP) in human biopsies obtained 6 months after SFA. Cylindrical biopsies, harvested 6 months after SFA, were processed for immunohistochemical analysis of sawed hard tissue sections using primary antibodies specific to osteocalcin (OC), collagen type I (Col I), bone sialoprotein (BSP), and alkaline phosphatase (ALP). Furthermore, the bone and particle area fraction were measured histomorphometrically in all biopsies in order to characterize bone formation and degradation of the bone grafting materials. Both biomaterials facilitated osteoblast differentiation and bone regeneration of resorbed alveolar ridges, resulting in sufficient bone formation for supporting dental implants. Si-CAOP specimens displayed more advanced bone formation and significantly greater particle degradation than TCP sites. This was accompanied by significantly higher expression of OC, Col I, BSP, and ALP in the osteoid in contact with the degrading particles. These promising results indicate that the Si-CAOP material has great potential use for SFA in humans, thereby confirming its superiority to $\beta$-TCP. A prospective study involving a larger patient number, a split-mouth design and analysis of angiogenic properties and of cone-beam CT-data for assessing the volume stability of the augmented area is warranted to further confirm the high osteogenic capacity of Si-CAOP for orofacial bone regeneration.
\end{abstract}

Keywords: Sinus floor augmentation; Bone substitutes; Calcium alkali orthophosphates; Osteogenesis; Osteogenic marker expression; Implant dentistry 